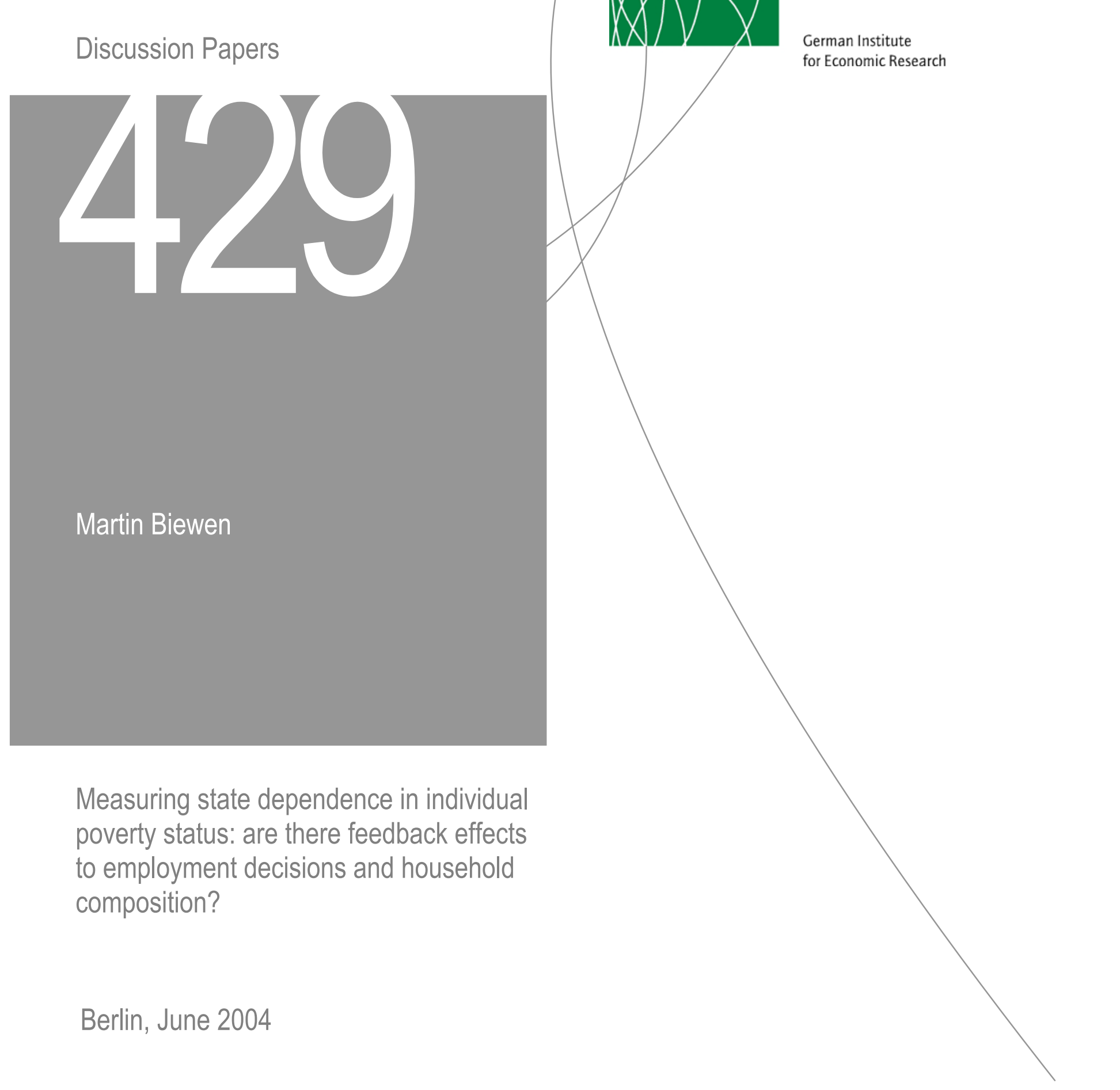


Opinions expressed in this paper are those of the author and do not necessarily reflect views of the Institute.

DIW Berlin

German Institute

for Economic Research

Königin-Luise-Str. 5

14195 Berlin,

Germany

Phone +49-30-897 89-0

Fax $\quad+49-30-89789-200$

www.diw.de

ISSN 1619-4535 


\title{
Measuring state dependence in individual poverty sta- tus: are there feedback effects to employment decisions and household composition? ${ }^{1}$
}

\author{
Martin Biewen \\ University of Mannheim
}

IZA, Bonn

DIW Berlin

This version: 30 April 2004

\begin{abstract}
Using a sample of prime-aged men from the German Socio-Economic Panel (GSOEP), this paper examines the effects of past poverty experience on future poverty status, future employment status and household composition. The empirical results suggest that even after controlling for observed and unobserved characteristics, past poverty experience increases the poverty risk of future periods. Moreover, there is evidence that experiencing poverty has a negative effect on future employment behaviour and on household cohesion. Apart from its economic significance, the existence of such feedback effects is interesting from an econometric point of view, as they represent a violation of the strict exogeneity assumption, which is usually invoked in estimating dynamic qualitative response models with unobserved heterogeneity.
\end{abstract}

JEL-Classification: C33, D31, I32, J12

Keywords: Poverty persistence, dynamic binary response models, correlated random effects, initital conditions, strict exogeneity

Correspondence:

Martin Biewen, Department of Economics, University of Mannheim, Verfügungsgebäude L7, 3-5, 68131 Mannheim, Germany, Fax: +49-621-1811841, biewen@rhein.vwl.uni-mannheim.de

\footnotetext{
${ }^{1}$ Financial support of the Deutsche Forschungsgemeinschaft (DFG) is gratefully acknowledged. I would like to thank Bernd Fitzenberger, Stefan Hoderlein, Enno Mammen, and seminar participants in Mannheim for helpful comments. The data used in this paper were made available by the German Socio Economic Panel Study (GSOEP) at the German Institute for Economic Research (DIW), Berlin.
} 


\section{Introduction}

It is a well-established finding that individuals who experience poverty are more likely to experience poverty in future periods. In principle, such a relationship may be due to two fundamentally different mechanisms. The first possibility is that individuals who are poor in one period are so because they have characteristics that make them particularly povertyprone. This might be observed characteristics such as low endowments of human capital, unemployment, health problems or difficult living arrangements, or typically unobserved factors such as low intelligence, lack of abilities, low levels of motivation or unfavorable general attitudes. To the extent that these characteristics persist over time, they will also increase the poverty risk of future periods, creating a spurious relationship between current and future poverty.

The alternative possibility is that the poverty experience of one period has a genuine causal effect on future poverty (this is usually called the state dependence effect). There are a number of different mechanisms that might explain such a causal effect. A first example is that low income may be associated with adverse incentives which make it unworthwhile for the individual to take up a job if unemployed, or even to keep a low-paid job if employed (the so-called poverty trap). This is a realistic possibility in countries with a minimum-income guarantee such as Germany, where unemployed individuals near the minimum-income level face excessive marginal tax and transfer burdens when they increase their earnings over this minimum level. Another channel through which a poverty experience may increase the risk of future poverty is if it is connected to processes of demoralization, loss of motivation or depreciation of human capital, which make it less likely that the individual takes up a job if unemployed, or which may lead to a series of low quality jobs or unstable employment, increasing in turn the risk of remaining in or returning to poverty. A similar mechanism is at work if the experience of low income or the feeling of social exclusion leads to problems with alcohol or other drugs, or more generally to health problems, which typically make it difficult to remain gainfully employed. In a similar way, a poverty experience may also be associated with a change in the living milieu and an increase in 'bad' contacts which may have detrimental effects on the quality of job opportunities, or which may lead to participation in a 'culture of dependency', where welfare receipt is the accepted way of living. Finally, having to live on low financial means may strain marriages or cohabitative relationships and 
possibly increase the probability of a household split, which in turn will lead to higher poverty risks if household economies-of-scale are destroyed or if work incentives are diminished by maintenance payments.

The channels through which possible causal effects from current to future poverty work are also interesting from an econometric point of view. If effects of poverty on future poverty are investigated using dynamic qualitative response models, and if these effects are mediated through feedback between poverty status and observed variables included as regressors in the model, then this represents a violation of the strict exogeneity assumption usually invoked in estimating such models (see Arellano/Honoré (2001) and Honoré (2002) for an overview). In the present context this concerns in particular feedback from poverty to employment status, and from poverty to household composition.

The distinction between poverty persistence due to individual heterogeneity as opposed to poverty persistence due to a causal effect of current on future poverty has important policy implications. If poverty causes new poverty independently of other causes, then povertyfighting policies will have a much more profound impact, as not only current poverty but also future poverty is avoided. On the other hand, if there is evidence that poverty has a tendency to reproduce itself then existing policies will have to be checked to what extent they might be a part of such a mechanism. For example, it will have to be examined if adverse incentives prevent low income individuals from taking up a job or whether generous welfare payments lead to a 'culture of dependency'.

Using data from the German Socio-Economic Panel (GSOEP), the aim of this paper is to examine the causal effects of current on future poverty, explicitly considering possible feedback between poverty status, employment status and household composition. After reviewing dynamic binary response models which are in principle suited to study the question, and after discussing the role of the strict exogeneity assumption, a joint dynamic model of poverty, employment status and household composition based on an idea by Wooldridge (2000) is developed and estimated. The results suggest that (i) even after controlling for differently specified observed and unobserved individual heterogeneity there are causal effects from current to future poverty, that (ii) experiencing poverty decreases employment probabilities and increases the probability of living in a one-person-household in future periods, and that (iii), in the presence of such feedback effects, models based on the strict exogeneity 
assumption may yield biased estimates of state dependence effect.

The rest of the paper is organized as follows. In section 2, some related literature is discussed. Section 3 reviews dynamic binary response models based on the strict exogeneity assumption and develops a model explicitly allowing for feedback effects. Section 4 discusses the data used to estimate this model. The estimation results are presented in section 5 , while section 6 concludes the paper.

\section{Related literature}

Starting with Heckman $(1978,1981 \mathrm{a}, 1981 b)$ researchers have been asking the question whether observed persistence in economic phenomena is due to underlying differences in individual characteristics or due to genuine causal effects of past on future outcomes. A prominent example is the question of whether past unemployment causes future unemployment as studied for example by Flaig et al. (1993), Mühleisen/Zimmermann (1994) and Arulampalam et al. (2000). Another example is persistence in low pay, which has been considered, among others, by Stewart/Swaffield (1999), Weber (2002) and Stewart (2004). In most of the cited articles, dynamic discrete choice models with unobserved heterogeneity based on the strict exogeneity assumption are used to distinguish the effects of state dependence from observed and unobserved heterogeneity. The assumption of no feedback from the dependent variable on future values of the explanatory variables seems less problematic in these examples as the latter variables are usually individual characteristics such as age or educational qualifications that will not be altered by past outcomes of employment status or wages. ${ }^{2}$

The assumption of no feedback is much more problematic if one considers persistence in phenomena such as poverty or welfare participation which depend on household variables and employment decisions. ${ }^{3}$ Recent examples where models based on the strict exogeneity assumption are used to study state dependence in this context are Chay/Hyslop (2000),

\footnotetext{
${ }^{2}$ More problematic are marital status and the number of children, as one might expect, for example, negative effects of low wages on marriage stability and fertility.

${ }^{3}$ Another example where allowing no feedback from the dependent variable to future explanatory variables seems restrictive is health persistence as studied by Contoyannis et al. (2004). In their econometric specification, they implicitly assume that current health has no effects on future income or household composition.
} 
Poggi (2003) and Gong (2004). For example, Chay/Hyslop (2000) fit a number of alternative dynamic binary response models to labour force and welfare participation behaviour of women in the United States. In their case, the inclusion of marriage status and the number of children in a model for welfare participation might be problematic as past welfare participation may have effects on future marital status and fertility. In a similar way, Poggi (2003) studies persistence of social exclusion in Spain using a dynamic random effects probit model. Again, the inclusion of household type and employment dummies as regressors might be problematic as experiencing social exclusion may lead to changes in living arrangements or employment status. Finally, estimating a three-state dynamic multinomial logit model with random effects, Gong (2004) examines transition patterns for the welfare reliance of low income mothers in Australia. In his example, the no-feedback assumption may also be violated because some of the variables included as regressors, in particular the number of children, whether the woman in question lives with a partner, and whether this partner also relies on an income support payment, might depend on past welfare reliance.

A different approach to estimating state dependence effects in low income transitions is taken by Cappellari/Jenkins (2002, 2003). They essentially adopt a pooled estimation strategy which circumvents the strict exogeneity assumption. If one is not interested in the nature and the direction of the possible feedback effects, the pooled approach is a valid method to examine state dependence effects in the presence of unobserved heterogeneity. Besides a number of advantages, it also has some disadvantages which will be discussed in more detail below.

The present paper is also closely related to attempts of modelling poverty transitions in a more structural way undertaken by Burgess/Propper (1998), Burgess et al. (2002) and Aassve et al. (2004). In these papers, poverty transitions are modelled as the result of underlying transitions in economic and demographic variables such as employment, family union and child bearing decisions, emphasizing their possible interrelatedness through optimizing behaviour. Such an approach is in principle capable of incorporating feedback effects of past poverty status on future poverty, employment behaviour and household composition. However, a direct econometric implementation including unobserved heterogeneity and correlation of errors across the many processes considered seems difficult and leads to similar econometric problems as addressed by the strict exogeneity assumption. For example, in 
their empirical application Aassve et al. (2004) only model employment, family union and childbearing decisions as being jointly determined. Income and poverty status are then defined as a function of these variables. Feedback effects of past income on employment, family union and childbearing decisions, and direct effects of past income on future income (state dependence) are not allowed.

An alternative strategy to examining the effects of past on future outcomes is the duration or hazard framework (see e.g. Lancaster (1990)). In the presence of unobserved heterogeneity, the estimation of hazard models with time-varying regressors may also require a variant of the strict exogeneity assumption, ruling out certain relationships between the dependent variable and future outcomes of the time-varying regressors (see e.g. Wooldridge (2002b), section 20.4.3). This is also true of the simultaneous equations hazard approach of Lillard (1993), which aims at modelling the dynamic interrelationship between two or more duration variables. Even in this approach, it is implicitly assumed that the current hazard rate of a given process is unrelated to future values of all the variables used as its regressors (which may include present or past values of the other processes). In a recent application of Lillard's framework, Fitzgerald/Ribar (2003) jointly model transitions in and out of welfare participation and female household headship. In their example, this assumption amounts to assuming, for example, that the hazard of moving into female headship is unrelated to future welfare participation.

\section{Methods}

\subsection{Random effects estimation}

The first approach to modelling the dynamics of individual poverty status considered in this paper is a dynamic random effects probit model (see e.g. Wooldridge (2002a)). If $y_{i t}$ denotes poverty status of individual $i=1 \ldots N$ in period $t=1 \ldots T$ then

$$
y_{i t}=1\left\{\theta_{1} y_{i t-1}+\theta_{2} z_{i t}+c_{i}+e_{i t} \geq 0\right\}
$$

$(1\{\cdot\}$ is the indicator function) describes the evolution of poverty conditional on $i$ 's poverty status in the previous period, a vector of exogenous variables $z_{i t}$ and two unobservables $c_{i}$ 
and $e_{i t}$. The individual-specific term $c_{i}$ stands for all unobserved determinants of poverty that are time-invariant for a given individual (in the sense that they do not change over the sample period). In the poverty context these might be factors such as intelligence, ability, motivation or general attitudes. The residual variation $e_{i t}$ is idiosyncratic, and is assumed to follow a standard normal distribution, i.e. $e_{i t} \sim \mathcal{N}(0,1)$.

Two issues need to be addressed when modelling $c_{i t}$. Firstly, unobserved factors such as the ones mentioned above are likely to be correlated with the observed variables $z_{i t}$. For example, intelligence may be correlated with the human capital acquired by the individual, and the person's motivation may be related to his or her employment status. Secondly, $y_{i 0}$, i.e. the individual's poverty status in the initial period, may also be correlated with the factors captured by $c_{i t}$, as e.g. low intelligence or a lack of abilities will contribute to the risk of being poor in $t=0$.

Both of these aspects can be addressed by modelling the individual-specific term as

$$
c_{i}=\alpha_{0}+\alpha_{1} y_{i 0}+\alpha_{2} \bar{z}_{i}+a_{i}
$$

where $\bar{z}_{i}=T^{-1} \sum_{t=1}^{T} z_{i t}$ denotes the time-average of the observed variables $z_{i t}$ and $a_{i} \sim$ $\mathcal{N}\left(0, \sigma_{a}^{2}\right) \cdot{ }^{4}$

In order to estimate the model by conditional maximum-likelihood methods, one has to make the crucial assumption that

$$
P\left(y_{i t}=1 \mid z_{i}, y_{i t-1}, y_{i t-2}, \ldots, y_{i 0}, c_{i}\right)=P\left(y_{i t}=1 \mid z_{i t}, y_{i t-1}, c_{i}\right)
$$

(strict exogeneity of $z_{i}$, where $z_{i}$ summarizes the exogenous information $\left(z_{i 1}, \ldots, z_{i T}\right)$ ). This assumption means that conditional on poverty status in the previous period and conditional on the unobserved individual-specific characteristics $c_{i}$, poverty in period $t$ must not be related to the value of the explanatory variables in past or in future periods. This requires in particular that there must not be any feedback from poverty in period $t$ to future values of the explanatory variables. In the given context, this is likely to be unrealistic, as experiencing poverty in one period may possibly influence employment decisions or the household

\footnotetext{
${ }^{4}$ See Wooldridge (2002a). The usual way to account for the correlation between the initial condition $y_{i 0}$ and the individual specific effect is to model the distribution of $y_{i 0}$ given $c_{i}$ and the exogenous information $\left(z_{i 1}, \ldots, z_{i T}\right)$ (see Heckman (1981c)). Wooldridge (2002a)'s approach serves the same purpose but is much simpler.
} 
composition in future periods. We will relax the assumption of strict exogeneity and consider these cases in more detail below. In addition, (3) also assumes that only the first lag $y_{i t-1}$ is relevant for poverty in period $t$.

Assumption (3) can then be used to obtain the density of $\left(y_{i 1}, \ldots, y_{i T}\right)$ conditional on the exogenous variables $z_{i}$ and on the individual effect $c_{i}$

$$
f\left(y_{i 1}, \ldots, y_{i T} \mid z_{i}, c_{i}, \theta\right)=\prod_{t=1}^{T} f\left(y_{i t} \mid z_{i t}, y_{i t-1}, c_{i}, \theta\right) .
$$

As a next step, the unobserved term $c_{i}$ has to be integrated out

$$
\begin{aligned}
f_{i}(\theta, \alpha) & =f\left(y_{i 1}, \ldots, y_{i T} \mid z_{i}, y_{i 0}, \theta\right) \\
& =\int_{\mathbb{R}}\left[\prod_{t=1}^{T} f\left(y_{i t} \mid z_{i t}, y_{i t-1}, c, \theta\right)\right] h\left(c \mid z_{i}, y_{i 0}, \alpha\right) d c
\end{aligned}
$$

which is not difficult because $c_{i} \sim \mathcal{N}\left(\alpha_{0}+\alpha_{1} y_{i 0}+\alpha_{2} \bar{z}_{i}, \sigma_{a}^{2}\right)$. Assuming in addition that (1) follows a probit model yields

$$
f_{i}(\theta, \alpha)=\int_{\mathbb{R}}\left[\prod_{t=1}^{T} \Phi\left(\left(2 y_{i t}-1\right)\left(\theta_{1} y_{i t-1}+\theta_{2} z_{i t}+c\right)\right)\right] h\left(c \mid z_{i}, y_{i 0}, \alpha\right) d c
$$

where the integral can be evaluated using Gauss-Hermite quadrature (see Stroud/Secrest (1966) or Butler/Moffit (1982)). Estimates of $\theta=\left(\theta_{1}, \theta_{2}\right)$ and $\alpha=\left(\alpha_{1}, \alpha_{2}\right)$ can then be obtained by standard conditional maximum-likelihood methods, i.e. by maximizing $\log L(\theta, \alpha)=\sum_{i=1}^{N} \log f_{i}(\theta, \alpha)$ (see e.g. Wooldridge $\left.(2002 \mathrm{~b})\right)$.

\subsection{Fixed effects estimation}

One drawback of the correlated random effects model of the preceding section is that it assumes a rather specific relationship between the explanatory variables $z_{i}$ and the unobserved effect $c_{i}$. Although this relationship does not necessarily have to be specified as above, i.e. as a linear function of the time averages $\bar{z}_{i}$ (this has been done to save degrees of freedom), it could be restrictive in the given context. In order to avoid this restriction, a fixed effects logit approach can be employed (see Honoré/Kyriazidou (2000)).

For the dynamic fixed effects logit model, it is assumed that

$$
y_{i t}=1\left\{\theta_{1} y_{i t-1}+\theta_{2} z_{i t}+c_{i}+e_{i t} \geq 0\right\}
$$


as before, but with

$$
P\left(y_{i t}=1 \mid z_{i}, y_{i t-1}, c_{i}\right)=P\left(y_{i t}=1 \mid z_{i t}, y_{i t-1}, c_{i}\right)=\frac{\exp \left(\theta_{1} y_{i t-1}+\theta_{2} z_{i t}+c_{i}\right)}{1+\exp \left(\theta_{1} y_{i t-1}+\theta_{2} z_{i t}+c_{i}\right)} .
$$

The first equation in (8) is the strict exogeneity assumption again, which rules out feedback from the current poverty status to future values of the explanatory variables, while the second equation implies that the $e_{i t}$ 's follow an i.i.d. logistic distribution, independent of $z_{i}, c_{i}$ and $y_{i 0}$. In addition, it is assumed that the initial observation $y_{i 0}$ has an arbitrary probability distribution given $z_{i}$ and $c_{i}$

$$
P\left(y_{i 0}=1 \mid z_{i}, c_{i}\right)=p_{0}\left(z_{i}, c_{i}\right)
$$

To estimate this model, Honoré/Kyriazidou (2000) observed that for observations $1 \leq$ $t<s \leq T-1$

$$
P\left(y_{i t}=1, y_{i s}=0 \mid z_{i}, c_{i}, y_{i t}+y_{i s}=1, z_{i t+1}=z_{i s+1}, y_{i t-1}, y_{i s-1}, y_{i t+1}, y_{i s+1}\right)
$$

is independent of $c_{i}$ and that it can be calculated as

$$
p_{t s}(\theta)=\frac{\exp \left(\theta_{1}\left(y_{i t-1}-y_{i s+1}\right)+\theta_{1}\left(y_{i t+1}-y_{i s-1}\right) 1\{s-t>1\}+\theta_{2}\left(z_{i t}-z_{i s}\right)\right)}{1+\exp \left(\theta_{1}\left(y_{i t-1}-y_{i s+1}\right)+\theta_{1}\left(y_{i t+1}-y_{i s-1}\right) 1\{s-t>1\}+\theta_{2}\left(z_{i t}-z_{i s}\right)\right)} .
$$

It follows that

$$
P\left(y_{i t}=0, y_{i s}=1 \mid z_{i}, c_{i}, y_{i t}+y_{i s}=1, z_{i t+1}=z_{i s+1}, y_{i t-1}, y_{i s-1}, y_{i t+1}, y_{i s+1}\right)=1-p_{t s}(\theta)
$$

and that both

$$
P\left(y_{i t}=0, y_{i s}=0 \mid z_{i}, c_{i}, y_{i t}+y_{i s}=1, z_{i t+1}=z_{i s+1}, y_{i t-1}, y_{i s-1}, y_{i t+1}, y_{i s+1}\right)=0
$$

and

$$
P\left(y_{i t}=1, y_{i s}=1 \mid z_{i}, c_{i}, y_{i t}+y_{i s}=1, z_{i t+1}=z_{i s+1}, y_{i t-1}, y_{i s-1}, y_{i t+1}, y_{i s+1}\right)=0 \text {. }
$$

The model parameters $\theta=\left(\theta_{1}, \theta_{2}\right)$ can therefore be estimated by maximizing

$$
\sum_{i=1}^{N}\left[\sum_{1 \leq t<s \leq T-1} 1\left\{y_{i t}+y_{i s}=1\right\} 1\left\{z_{i t+1}=z_{i s+1}\right\} \log \left\{p_{t s}(\theta)^{y_{i t}}\left(1-p_{t s}(\theta)\right)^{1-y_{i t}}\right\}\right] .
$$


Note that (15) is as a standard problem of M-estimation (for details on M-estimation and on how an estimate of the variance-covariance matrix of the parameters can be computed, see e.g. Wooldridge (2002b)).

A difficulty of the fixed effect estimator is that typically only very few observations contribute identifying information. Firstly, only pairs of observations $t, s$ contribute to the criterion function where at the same time the poverty status changes, i.e. $y_{i t}+y_{i s}=1$, and where the vector of exogenous characteristics in the period after $t$ and $s$ is the same, i.e. $z_{i t+1}=z_{i s+1}$. Secondly, from (11), $\theta_{1}$ and $\theta_{2}$ can only be identified if there are observations for which in addition $y_{i t-1} \neq y_{i s+1}$ (or $y_{i t+1} \neq y_{i s-1}$ for $s-t>1$ ) and $z_{i t} \neq z_{i s}$. Together with the first requirement, the latter condition requires in particular that for their effects to be identified, the exogenous variables have to change over time, but not too fast. If the $z_{i t}$ are continuous, the strong equality constraints may be relaxed in a local smoothing procedure (see Honoré/Kyriazidou (2000)). Unfortunately, this is not an option here as most of the covariates (e.g. employment status and household composition) are of a discrete nature.

\subsection{Pooled estimation}

Both the random effects and the fixed effects approach are based on the strict exogeneity assumption, which may be questionable in the given context. A simple (but inefficient) alternative that avoids the strict exogeneity assumption is a pooled estimator (see e.g Wooldridge $(2002 b))$. Let

$$
y_{i t}=1\left\{\theta_{1} y_{i t-1}+\theta_{2} z_{i t}+c_{i}+e_{i t} \geq 0\right\}
$$

with

$$
c_{i}=\alpha_{0}+\alpha_{1} y_{i 0}+\alpha_{2} \bar{z}_{i}+a_{i}
$$

and $e_{i t} \sim \mathcal{N}(0,1), a_{i} \sim \mathcal{N}\left(0, \sigma_{a}^{2}\right)$ as in the random effects model. Multiplying the original parameters by $\operatorname{var}\left(a_{i}+e_{i t}\right)^{-1 / 2}=\left(1+\sigma_{a}^{2}\right)^{-1 / 2}$ implies that

$$
y_{i t}=1\left\{\theta_{1}^{a} y_{i t-1}+\theta_{2}^{a} z_{i t}+\alpha_{0}^{a}+\alpha_{1}^{a} y_{i 0}+\alpha_{2}^{a} \bar{z}_{i}+\frac{a_{i}+e_{i t}}{\sqrt{1+\sigma_{a}^{2}}} \geq 0\right\}
$$


can be described by a simple probit model with parameters $\theta_{1}^{a}, \theta_{2}^{a}, \alpha_{0}^{a}, \alpha_{1}^{a}, \alpha_{2}^{a}$, where superscript $a$ denotes the original parameters multiplied by $\left(1+\sigma_{a}^{2}\right)^{-1 / 2}$. The parameters $\theta_{1}^{a}, \theta_{2}^{a}, \alpha_{0}^{a}, \alpha_{1}^{a}, \alpha_{2}^{a}$ can therefore be estimated by running a probit model on the pooled observations (however, standard errors have to be adjusted to account for the fact that observations are dependent at the level of the individual.) An important drawback of the pooled approach is that the amount of unobserved heterogeneity $\sigma_{a}^{2}$ cannot be determined.

The reason why the pooled estimator is able to consistently estimate the state dependence parameter $\theta_{1}$ even in the presence of explanatory variables that are not strictly exogenous is that it makes no assumption on the joint distribution of $\left(y_{i 1}, \ldots, y_{i T}\right)$. If $\delta^{a, *}=\left(\theta_{1}^{a, *}, \theta_{2}^{a, *}, \alpha_{0}^{a, *}, \alpha_{1}^{a, *}, \alpha_{2}^{a, *}\right)$ are the true parameters and $f\left(y_{i t} \mid z_{i t}, y_{i t-1}, \delta^{a}\right)$ is the correctly specified conditional density of $y_{i t}$, then $\delta^{a, *}$ maximizes $E\left(\log f\left(y_{i t} \mid z_{i t}, y_{i t-1}, \delta^{a}\right)\right)$ for all $t$ (this follows from the Kullback-Leibler information inequality) and therefore $E\left[\sum_{t=1}^{T} \log f\left(y_{i t} \mid z_{i t}, y_{i t-1}, \delta^{a}\right)\right]$. This implies that maximizing $N^{-1} \sum_{i=1}^{N}\left[\sum_{t=1}^{T} \log f\left(y_{i t} \mid z_{i t}, y_{i t-1}, \delta^{a}\right)\right]$ yields consistent estimators of $\delta^{a, *}$ (see Wooldridge $(2002 b))$.

\subsection{A Model with feedback effects}

If there are feedback effects, the use of the dynamic random effects probit model as well as that of the fixed effects logit model is questionable. While the pooled estimator is in principle capable of identifying the state dependence parameter even in the presence of feedback effects, it will not be informative with respect to the existence and the direction of these feedback effects. Moreover, it is inefficient and cannot identify the share of unobserved heterogeneity $\sigma_{a}^{2} /\left(1+\sigma_{a}^{2}\right)$. The aim of this section is therefore to develop an econometric model which explicitly allows for feedback effects from poverty status to future employment decisions and household composition. The model will not only provide consistent estimates of the state dependence effect and the amount of unobserved heterogeneity, it will also shed light on the existence and direction of possible feedback effects, disclosing possible additional channels of causal transmission from past to future poverty.

Let $^{5} y_{i t}$ denote individual poverty status as before, and let $w_{i t}$ and $v_{i t}$ indicate whether

\footnotetext{
${ }^{5}$ The following is based on the general framework described in Wooldridge (2000).
} 
individual $i$ is employed and whether he (the empirical analysis will focus on prime-age males only) is living together with other persons. This will be a partner and possibly children in most cases. Then, under assumptions analogous to those made in the case of the random effects probit model, the joint density of $y_{i 1}, \ldots, y_{i T}, w_{i 1}, \ldots, w_{i T}, v_{i 1}, \ldots, v_{i T}$ given exogenous variables $z_{i}$, initial values $y_{i 0}, w_{i 0}, v_{i 0}$ and an individual-specific effect $c_{i}$ can be written as

$$
\begin{aligned}
f\left(y_{i 1}, \ldots, y_{i T}, w_{i 1}, \ldots, w_{i T}, v_{i 1}, \ldots, v_{i T} \mid z_{i}, y_{i 0}, w_{i 0}, v_{i 0}, c_{i}, \theta, \gamma, \beta\right) & \\
=\prod_{t=1}^{T} \quad & f\left(y_{i t} \mid z_{i t}, w_{i t}, v_{i t}, y_{i t-1}, w_{i t-1}, v_{i t-1}, c_{i}, \theta\right) \\
& \cdot f\left(w_{i t} \mid z_{i t}, v_{i t}, y_{i t-1}, w_{i t-1}, v_{i t-1}, c_{i}, \gamma\right) \\
& \cdot f\left(v_{i t} \mid z_{i t}, y_{i t-1}, w_{i t-1}, v_{i t-1}, c_{i}, \beta\right) \\
=\prod_{t=1}^{T} \quad & \Phi\left(\left(2 y_{i t}-1\right)\left(\theta_{1} z_{i t}+\theta_{2} w_{i t}+\theta_{3} v_{i t}+\theta_{4} y_{i t-1}+\theta_{5} w_{i t-1}+\theta_{6} v_{i t-1}+c_{i}\right)\right) \\
& \cdot \Phi\left(\left(2 w_{i t}-1\right)\left(\gamma_{1} z_{i t}+\gamma_{2} v_{i t}+\gamma_{3} y_{i t-1}+\gamma_{4} w_{i t-1}+\gamma_{5} v_{i t-1}+\gamma_{6} c_{i}\right)\right) \\
& \cdot \Phi\left(\left(2 v_{i t}-1\right)\left(\beta_{1} z_{i t}+\beta_{2} y_{i t-1}+\beta_{3} w_{i t-1}+\beta_{4} v_{i t-1}+\beta_{5} c_{i}\right)\right)
\end{aligned}
$$

where the individual-specific effect now also includes the influence of the initial values of employment status and living arrangements, i.e.

$$
c_{i}=\alpha_{0}+\alpha_{1} y_{i 0}+\alpha_{2} w_{i 0}+\alpha_{3} v_{i 0}+\alpha_{4} \bar{z}_{i}+a_{i}
$$

Note that the additional equations for employment status (21) and household composition (22) also include lagged poverty experience, capturing possible feedback effects from poverty to employment status and to whether the individual lives alone. Such feedback effects may reflect detrimental effects of poverty on the morale of the individual, leading to lower employment probabilities, or on the stability of marriages or cohabitative relationships, increasing the probability of a household split. A negative effect of poverty on employment status may also be the result of adverse incentives (the so-called poverty trap), where high marginal tax and transfer burdens prevent individuals from taking up a job.

Note that the three equations are not truly simultaneous, as e.g. current poverty does not enter the employment equation. It is well known that truly simultaneous systems of qualitative outcomes are logically inconsistent (see Maddala (1983), section 5.7). Equations (21) and (22) also control for state dependence effects in employment behaviour and living arrangements, and for possible correlations between employment status, living arrangements 
and the unobserved time-invariant determinants of poverty status $c_{i}$. Estimation of the model is analogous to the single-equation random effects probit model, i.e. the individual-specific effect $c_{i}$ has to be integrated out ((19) takes the place of (4) in (5)).

A limitation of the model is that it does not allow for serial correlation in the idiosyncratic error terms. Serial correlation in dynamic binary response models can in principle be taken account of using simulation methods (see Hajivassiliou/Ruud (1994) and Hyslop (1999)). However, given the multiple equations structure of the model, it would be exceedingly difficult to model serial correlation in the given case. Moreover, the results for welfare participation in Chay/Hyslop (2000) suggest that controlling for serial correlation makes little difference in the given context. Interestingly, the pooled estimator is also robust to serial correlation. A comparison of the pooled estimates with the ones of the feedback model (see below) also indicates that serial correlation is not an important issue in the application considered here.

\subsection{Other methods}

Alternative methods for estimating dynamic binary choice models with regressors that potentially violate the strict exogeneity assumption have recently been proposed by Honoré/Lewbel (2002) and Arellano/Carrasco (2003). In Honoré/Lewbel (2002), estimation is based on the assumption that there exists a continuous regressor that is independent of the individual specific effect and the error term, conditional on the other regressors. As both assumptions are clearly violated in the given context (all regressors are discrete and likely to be correlated with the individual effect), this estimator is not suited for the application pursued here. Arellano/Carrasco (2003) propose a semi-parametric estimator based on the cell-averages of all possible time-paths of the regressors up to a given period. However, in an application with a moderate to large number of regressors and many time periods as it is considered here, this usually leads to a large number of empty cells and requires the use of trimming methods. ${ }^{6}$ Their approach is therefore not further pursued here.

\footnotetext{
${ }^{6}$ In their empirical illustration, Arellano/Carrasco (2003) consider an example with only two regressors.
} 


\subsection{Estimating average partial effects}

In order to assess the magnitude of state dependence and feedback effects, it is useful to calculate average partial effects. In the given context, average partial effects show the impact of a change in an explanatory variable on one of the endogenous variables, i.e. on the risk of experiencing poverty, the probability of being employed or the probability of living together with other persons, averaged over the distribution of the other characteristics in the population. For example, in the model with feedback effects the average partial effect of lagged poverty status (the state dependence effect), is given by

$$
\begin{aligned}
A P E= & E\left[P\left(y_{i t}=1 \mid z_{i}, w_{i t}, v_{i t}, y_{t-1}=1, w_{i t-1}, v_{i t-1}, y_{i 0}, w_{i 0}, v_{i 0}\right)\right. \\
& \left.-P\left(y_{i t}=1 \mid z_{i}, w_{i t}, v_{i t}, y_{t-1}=0, w_{i t-1}, v_{i t-1}, y_{i 0}, w_{i 0}, v_{i 0}\right)\right]
\end{aligned}
$$

where the expectation is over all characteristics indexed by $i$. This average partial effect can be consistently estimated by

$$
\begin{aligned}
\widehat{A P E}=\sum_{i=1}^{N}\left[\Phi \left(\hat{\theta}_{1}^{a} z_{i t}+\hat{\theta}_{2}^{a} w_{i t}+\right.\right. & \hat{\theta}_{3}^{a} v_{i t}+\hat{\theta}_{4}^{a} \cdot 1+\hat{\theta}_{5}^{a} w_{i t-1}+\hat{\theta}_{6}^{a} v_{i t-1} \\
& \left.+\hat{\alpha}_{0}^{a}+\hat{\alpha}_{1}^{a} y_{i 0}+\hat{\alpha}_{2}^{a} w_{i 0}+\hat{\alpha}_{3}^{a} v_{i 0}+\hat{\alpha}_{4}^{a} \bar{z}_{i}\right) \\
-\Phi\left(\hat{\theta}_{1}^{a} z_{i t}+\hat{\theta}_{2}^{a} w_{i t}\right. & +\hat{\theta}_{3}^{a} v_{i t}+\hat{\theta}_{4}^{a} \cdot 0+\hat{\theta}_{5}^{a} w_{i t-1}+\hat{\theta}_{6}^{a} v_{i t-1} \\
& \left.\left.+\hat{\alpha}_{0}^{a}+\hat{\alpha}_{1}^{a} y_{i 0}+\hat{\alpha}_{2}^{a} w_{i 0}+\hat{\alpha}_{3}^{a} v_{i 0}+\hat{\alpha}_{4}^{a} \bar{z}_{i}\right)\right]
\end{aligned}
$$

(superscript $a$ denotes again the original parameter estimates multiplied by $\left(1+\hat{\sigma}_{a}^{2}\right)^{-1 / 2}$ ).

Average partial effects in the single-equation random effects model and in the pooled model are estimated analogously. As the relationship between the explanatory variables $z_{i}$ and the individual effect $c_{i}$ remains unspecified in the fixed effects model, no partial average effects are available in this approach. This is a serious drawback of the fixed effect approach, as the magnitude of the state dependence effect (and that of other variables) cannot be determined (this was pointed out by Wooldridge (2002a)). 


\section{Data}

The empirical analysis in the following section is based on a sample taken from the German Socio-Economic Panel (GSOEP). The GSOEP is a representative panel study for Germany which was started in 1984 for West Germany and which was extended to East Germany after reunification of the country in 1990 (for a more detailed description, see SOEP Group (2001) and Haisken-DeNew/Frick (2003)). As most of the mechanisms discussed in the introduction apply to individuals who are in principle attached to the labour market, the analysis focuses on men aged between 18 and 65 years. The sample used here consists of 2427 East and West German men (including foreigners) and covers the years 1991 to 2001.

The variables that were treated as potentially being endogenous were individual poverty status, individual employment status and whether the individual lived in a one-personhousehold. Individual poverty status was derived from monthly net household income using a standard equivalence scale (the so-called OECD-scale, see e.g. Atkinson et al. (1995)). Applying this scale, household incomes were first divided by the square-root of household size. The result of this calculation was then attributed to each household member as a measure of his or her personal disposable income. Following a conventional standard for Germany, ${ }^{7}$ the poverty line was set to one half of the mean of these equivalized personal incomes in each year. The poverty line was calculated for the whole population (including children), while for the final sample only men aged 18 to 65 years were selected.

The definition of employment status included part-time work, although this concerned only a very small fraction of the sample. Household composition was captured by a binary variable indicating whether the individual lived alone or together with others, which was in most cases a partner (married or cohabiting) and possibly children. All other variables were treated as exogenous. These were in particular variables indicating the highest achieved educational qualifications, individual age, nationality, and whether the individual resided in East or in West Germany. With regard to the highest achieved educational qualifications, individuals were grouped into three categories. These were (i) individuals holding a university degree, (ii) individuals who had Abitur (equivalent to a high-school degree) or who underwent vocational training (Lehre), and (iii) all others.

\footnotetext{
${ }^{7}$ See e.g. Bundesministerium für Arbeit und Sozialordnung (2001).
} 
Although the model with feedback effects described in the previous section is identified without exclusion restrictions (due to its recursive structure), additional exogenous variables were included in the employment equation and in the equation for household composition. These were the regional vacancy ratio by federal state (Bundesland) and a dummy indicating whether the individual's last job was terminated by firm closure for the employment equation, and the share of prime-aged men living together with other persons (estimated from the sample) and a dummy for church attendance in the equation for household composition. The idea of the latter variables was to pick up societal trends in living arrangements (which are exogenous to the individual) and personal attitudes towards marriage. To the extent that these variables are exogenous, they provide additional variation for the identification of the dynamic interplay between poverty status and the other two endogenous variables.

\section{Empirical results}

The presentation of the empirical results proceeds in two steps. In a first step, the results of the single-equation models are presented and discussed. In a second step, these results are then contrasted with the estimates of the model with feedback effects.

The first column of table 1 shows the results for the single-equation correlated random effects model. The estimate for the state dependence effect is sizable and statistically significant. It suggests that even after controlling for differences in observed and unobserved characteristics, past poverty experience was connected to a higher future poverty risk. The effects of other characteristics were in line with prior expectations. For example, being gainfully employed significantly reduced the poverty risk in a given period. Living together with others also reduced the risk of being poor, but this effect was not as strong as that of being employed. An explanation for the latter effect is that individuals who live together with others benefit from household economies-of-scale and from the earnings of their partners or other household members.

- Table 1 about here -

As expected, having a university degree reduced the poverty risk, but this effect was 
not statistically significant. However, the estimates for the correlated part of the random effect (lower part of table 1) suggest that holding a university degree was connected to unobserved characteristics that strongly reduced the probability of being poor. In a similar way, unobserved characteristics connected to employment status were associated with lower poverty risks. The estimates in the upper part of table 1 further show the age profile of poverty. The poverty risk decreased with age, where again part of this effect was due to unobserved characteristics that were correlated with age (see lower part of the table). Finally, non-German nationality, living in East Germany and low income in the initial year 1991 were associated with higher poverty rates (as the characteristic nationality was practically timeinvariant, it was only included in the correlated part of the permanent effect).

The second column of table 1 show the corresponding results for the fixed effects model. Again, a statistically significant effect of past on future poverty is found. However, because of the unmodelled error variance in this model (the permanent individual effect $c_{i}$ and its variance was left unspecified), the magnitudes of the coefficients cannot be compared to the ones of the random effects model. It seems however that relative magnitudes are similar. Apart from the state dependence effect most of the estimates are rather imprecise, which stems from the fact that typically a very small number of observations identified the respective effect (see the discussion in section 3). For example, the effect of the East Germany dummy was identified by a mere thirteen observations that were contributed by only six individuals.

The last column of table 1 presents the results for the pooled model. Apart from the state dependence parameter, which was estimated about twice the size of that in the random effects model, the estimated effects are remarkably similar to the ones in the random effects model. (The coefficients of the two models are directly comparable since both models were normalized to have an error variance of one.) As discussed above, the estimates of the pooled model are a-priori more reliable than those of the random effects model as they are robust to a violation of the strict exogeneity assumption. The fact that the effects are so similar except for the one for state dependence might therefore indicate that the random effects estimate might be biased due to a violation of the no-feedback assumption.

This question can be addressed using the model with feedback. The estimation results of this model are shown in table 2. Column 1 of the table displays the coefficients for the poverty 
equation. As in the other models, there were strong and significant poverty-reducing effects of employment and of living together with others. The results also suggest a sizeable and significant state dependence effect, the magnitude of which even exceeded that of employment (the magnitude of the most interesting effects in terms of average partial effects are discussed in more detail below). There was also a negative (but smaller) effect of past employment on current poverty status, which indicates that even after controlling for current employment status, past unemployment had a detrimental effect on poverty risk, e.g. through worsening job quality or displaced workers penalties. The effects for the other variables show similar patterns as in the previous models, although the estimates seem less precise due to the larger number of estimated parameters.

- Table 2 about here -

The results for the employment equation are given in the second column of table 2 . They suggest that current household composition had a significant negative effect on employment in the sense that men who lived alone were less likely to be non-employed than those who lived together with others. A possible explanation is that those living alone cannot count on the financial support of other household members and therefore face stronger incentives to seek employment. The results for the employment equation also provide evidence for significant feedback from past poverty to future employment in the form of a strongly negative effect. This means that past poverty experiences diminished the probability of employment in future periods, which can be interpreted as evidence for a poverty trap. There was also a strong state dependence effect in employment behaviour, i.e. even after controlling for other characteristics, employment in one period led to higher employment probabilities in future periods. This confirms state dependence effects for unemployment found in other studies, see e.g. Mühleisen/Zimmermann (1993) and Flaig et al. (1993) for Germany or Arulampalam et al. (2000) for Britain.

The effects of the other variables on employment were in line with prior expectations. Higher educational qualifications were associated with higher employment probabilities and there was a concave age profile of employment. The decreasing employment probability of older men can be explained by the wide spread practice of early retirement in conjunction with an extended period of unemployment benefit receipt. East German men had lower 
employment probabilities than their West German counterparts, which was due to the much higher unemployment rate in East Germany. On the other hand, there was no additional explanatory power of the regional vacancy ratio (the number of vacancies divided by the number of unemployed, disaggregated by federal states). By contrast, the instrument of firm closure was highly significant. If the last job of the individual was terminated by firm closure, this significantly diminished the probability of employment in later periods. As firm closure is exogenous to the individual, inclusion of this variable shifts employment independently of the individual factors affecting poverty and thus provides additional variation to identify the effect of employment on poverty status. Finally, the results at the bottom of table 2 show that the unobserved individual-specific factors determining poverty were negatively related to the probability of being employed.

Column 3 of table 2 presents the corresponding results for household composition. Again, there was a statistically significant feedback effect of poverty on household composition. A poverty experience in one period led to a higher probability of living in a oneperson-household in the next period. This can be interpreted as evidence that low income increased the probability of a household-split. It is interesting to see that this was the effect of low income and not of unemployment, as the corresponding coefficient for employment was small and statistically insignificant. There was also a strong state dependence effect in household composition, which reflects the costs of household dissolution especially in the case of married couples. The age-profile of household composition was concave, with higher probabilities of living alone at young and at older ages. Living arrangements also seemed strongly related to societal trends, as the extremely large estimate for the effect of the aggregate living-with-others ratio suggests. Church attendance, which can be seen as a proxy for personal attitudes towards marriage, was also positively related to the probability of living with others, but the corresponding effect was much smaller than that of aggregate trends. Finally, there was a negative association between the the probability of living alone and the unobserved individual-specific determinants of poverty.

How large are these effects in percentage points? Table 3 presents the most interesting estimates expressed as average partial effects. According to the random effects estimates, being poor increased the poverty risk in the next period by roughly 8 percentage points. By contrast, the pooled model estimated this effect as approximately 24 percentage points. The 
pooled estimate was very similar to the estimate from the feedback model, which implied that poverty in the previous period increased the poverty risk in the next period by 22 percentage points. The fact that the pooled model and the model with feedback yielded very similar results and that these results were different from those of the potentially misspecified random effects model suggests that the random effects estimates were biased.

— Table 3 about here -

Table 3 also shows the aggregate transition probabilities, which can be interpreted as unconditional estimates without controlling for differences in observed or unobserved characteristics. According to these estimates, those poor in the previous period had a poverty risk that was 45 percentage points higher than that of individuals who were not poor in the previous period. In conjunction with the estimates of the pooled or the feedback model this implies that about half of poverty persistence was due to state dependence, while the other half was due to persistence in observed and unobserved characteristics.

The rest of the table shows the effects for the other variables. Being employed reduced the poverty risk by almost 14 percentage points and individuals who lived together with others had about six percentage points lower poverty rates than those who did not, other things being equal. Employment probabilities were reduced by two percentage points on average if the individual in question lived together with others, and by almost four percentage points if the individual was poor in the previous period. There was considerable state dependence in employment status, with individuals who were employed in the previous period having 17 percentage points higher employment probabilities than those who were not. Finally, poverty in the previous period reduced the probability of living together with others by one percentage point and increased it by roughly 88 percentage points if the individual already lived together with others in the previous period.

\section{Conclusion}

This paper considered the question of whether there are causal effects from past poverty experience on future poverty status, employment status and household composition. The 
results suggest that even after controlling for observed and unobserved differences, experiencing poverty was associated with an increased poverty risk in future periods. Moreover, there is evidence that experiencing poverty had a detrimental effect on future employment behaviour and household cohesion. The estimates thus provide evidence that poverty experiences can be associated with processes of demoralization, depreciation of human capital and with incentive problems, increasing the probability that individuals who become poor will remain so for an extended period of time. There is also evidence that poverty experiences may reduce household cohesion, indicating that low income strains marriages and cohabitative relationships. Future research should address in more detail through which channels these effects work and what their relative importance is.

The paper also highlighted the role of the strict exogeneity assumption in estimating dynamic binary response models with unobserved heterogeneity. The existence of feedback effects of poverty status on future values of the variables that are used to explain the poverty risk of a given period makes the use of models based on the strict exogeneity assumption questionable. Based on the framework of Wooldridge (2000), this paper therefore developed a dynamic model of poverty which explicitly allowed for such feedback effects. The estimation results suggest that in the presence of feedback effects, models based on the strict exogeneity assumption may yield biased results. 


\section{References}

Aassve, A., S. Burgess and C. Propper (2004): Modelling Poverty transitions as the outcome of employment, family union, and childbearing decisions in the United Kingdom, unpublished manuscript, University of Bristol.

Arellano, M. and B. Honoré (2001): Panel data models: some recent developments, in: Heckman, J.J. and E. Leamer (eds.), Handbook of Econometrics, Vol. 5, Amsterdam: Elsevier Science B.V., pp. 3229 - 3296.

Arellano, M. and R. Carrasco (2003): Binary choice panel data models with predetermined variables, Journal of Econometrics 115, pp. 125 - 157.

Arulampalam, W., Booth, A.L. and Taylor, M.P. (2000): Unemployment persistence, Oxford Economic Papers 52, pp. 24 - 50.

Bundesministerium für Arbeit und Sozialordnung (2001): Lebenslagen in Deutschland, Der erste Armuts- und Reichtumsbericht der Bundesregierung, Berlin.

Burgess, S. and C. Propper (1998): An economic model of household income dynamics, with an application to poverty dynamics among American women, CEPR Discussion Paper No. 1830, CEPR, London.

Burgess, S., C. Propper and M. Dickson (2002): The Analysis of Poverty Data with Endogenous Transitions, unpublished manuscript, University of Bristol.

Butler, J.S., and R. Moffit (1982): A Computationally Efficient Quadrature Procedure for the One-Factor Multinomial Probit Model, Econometrica 50, pp. 761 - 764.

Cappellari, L. and S.P. Jenkins (2002): Modelling low income transitions, ISER Working Papers 2002-8, Institute for Social and Economic Research, University of Essex, forthcoming in: Journal of Applied Econometrics.

Cappellari, L. and S.P. Jenkins (2003): Transitions between unemployment and low pay, unpublished manuscript, Institute for Social and Economic Research, University of Essex.

Chay, K.Y. and D.R. Hyslop (2000): Identification and Estimation of Dynamic Binary Re- 
sponse Models: Empirical Evidence using Alternative Approaches, University of California, Berkeley, forthcoming in: Journal of Econometrics.

Contoyannis, P., A.M. Jones and N. Rice (2004): Simulation-based inference in dynamic panel probit models: An application to health, Empirical Economics 29, pp. 49 - 77.

Fitzgerald, J.M. and D.C. Ribar (2003): Transitions in Welfare Participation and Female Headship, IZA Discussion Paper No. 895, Institute for the Study of Labor, Bonn.

Flaig, G., Licht, G. and V. Steiner (1993): Testing for state dependence effects in a dynamic model of male unemployment behaviour, in: Bunzel, H., Jensen, P. and N. WestergaardNielsen (eds.), Panel data and labour market dynamics, Amsterdam: North Holland.

Gong, X. (2004): Transition Patterns for the Welfare Reliance of Low Income Mothers in Australia, IZA Discussion Paper No. 1047, Institute for the Study of Labor, Bonn.

Haisken-DeNew, J.P. and J. Frick (eds.) (2003): Desk Top Companion to the German SocioEconomic Panel Study (GSOEP) - Version \%.0, Deutsches Institut für Wirtschaftsforschung (DIW), Berlin.

Hajivassiliou, V.A. and P.A. Ruud (1994): Classical Estimation Methods for LDV Models using Simulation, in: Engle, R.F. and D.L. McFadden, Handbook of Econometrics, Vol. 4, Amsterdam: Elsevier Science B.V., pp. 2383 - 2441.

Heckman, J. (1981a): Statistical models for discrete panel data, in: Manski, C., D. McFadden (eds.), Structural Analysis of Discrete Data with Econometric Applications, Cambridge, MA: MIT Press, pp. 114 - 178.

Heckman, J. (1981b): Heterogeneity and state dependence, in: Rosen, S. (ed.), Studies in Labor Markets, Chicago: University of Chicago Press.

Heckman, J. (1981c): The Incidental Parameter Problem and the Problem of Initial Conditions in Estimating a Discrete Time-Discrete Data Stochastic Process, in: Manski, C., D. McFadden (eds.), Structural Analysis of Discrete Data with Econometric Applications, Cambridge, MA: MIT Press, pp. 179 - 195.

Honoré, B.E. (2002): Nonlinear Models with Panel Data, Cemmap working paper 13/02, 
Institute for Fiscal Studies, London.

Honoré, B.E. and E. Kyriazidou (2000): Panel data discrete choice models with lagged dependent variables, Econometrica 68, pp. 839 - 874.

Honoré, B.E. and A. Lewbel (2000): Semiparametric binary choice panel data without strictly exogenous regressors, Econometrica 70, pp. 2053 - 2063.

Hyslop, D.R. (1999): State Dependence, Serial Correlation and Heterogeneity in Intertemporall Labor Force Participation Behavior of Married Women, Econometrica 67, pp. 1255 1294.

Lancaster, T. (1990): The Econometric Analysis of Transition Data, Cambridge: Cambridge University Press.

Maddala, G. (1983): Limited-Dependent and Qualitative Variables in Econometrics, Cambridge: Cambridge University Press.

Mühleisen, M. and K.F. Zimmermann (1994): A panel analysis of job changes and unemployment, European Economic Review 38, pp. 793 - 801.

Poggi, A. (2003): Does persistence of social exclusion exist in Spain? Working Paper No. 03.08, Departament d'Economia Aplicada, Universitat Autonoma de Barcelona.

SOEP Group (2001): The German Socio-Economic Panel (GSOEP) after more than 15 years, in: Holst, E., D.R. Lillard and T.A. DiPrete (eds.), Proceedings of the 2000 Fourth International Conference of German Socio-Economic Panel Study Users (GSOEP2000), Vierteljahreshefte zur Wirtschaftsforschung 70, pp. 7 - 14.

Stewart, M.B. and J.K. Swaffield (1999): Low Pay Dynamics and Transition Probabilities, Economica 66, pp. $23-42$.

Stewart, M.B. (2002): The Inter-related Dynamics of Unemployment and Low Pay, unpublished manuscript, University of Warwick.

Strout, A.H. and D. Secrest (1966): Gaussian quadrature formulas, Englewood Cliffs: Prentice Hall. 
Weber, A. (2002): State dependence and wage dynamics: a heterogenous Markov chain model for wage mobility in Austria, unpublished manuscript, Institute for Advanced Studies, Vienna.

Wooldridge (2000): A framework for estimating dynamic, unobserved effects panel data models with possible feedback to future explanatory variables, Economics Letters 68, pp. $245-250$.

Wooldridge (2002a): Simple solutions to the initial conditions problem in dynamic, nonlinear panel data models with unobserved heterogeneity, Cemmap Working Paper 18/02, Institute for Fiscal Studies, London.

Wooldridge (2002b): Econometric Analysis of Cross Section and Panel Data, Cambridge, MA: MIT-Press. 


\section{Tables}

Table 1. Single equation dynamic binary response models for individual poverty status (standard errors in parentheses)

\begin{tabular}{|c|c|c|c|c|c|c|}
\hline & \multicolumn{2}{|c|}{ random effects ${ }^{a}$} & \multicolumn{2}{|c|}{ fixed effects ${ }^{b}$} & \multicolumn{2}{|c|}{ pooled model ${ }^{c}$} \\
\hline \multicolumn{7}{|c|}{ Dependent variable: individual poverty status } \\
\hline lagged poverty status & 0.6582 & $(0.0500)$ & 1.2361 & $(0.1563)$ & 1.3956 & $(0.0567)$ \\
\hline employed & -0.7087 & $(0.0451)$ & -1.5849 & $(0.1979)$ & -0.7182 & $(0.0569)$ \\
\hline living w. others & -0.3694 & $(0.0882)$ & -0.7857 & $(0.3621)$ & -0.3134 & $(0.0945)$ \\
\hline university degree & -0.1543 & $(0.1401)$ & -0.7072 & $(0.7170)$ & -0.0911 & $(0.1433)$ \\
\hline Abitur or Lehre ${ }^{d}$ & 0.0123 & $(0.0754)$ & 0.3337 & $(0.4122)$ & 0.0167 & $(0.0720)$ \\
\hline $26-35$ years & -0.0538 & $(0.0778)$ & -0.1524 & $(0.3509)$ & -0.0263 & $(0.0887)$ \\
\hline $36-45$ years & -0.1075 & $(0.1009)$ & -0.1674 & $(0.4781)$ & -0.0350 & $(0.1081)$ \\
\hline $46-55$ years & -0.1839 & $(0.1280)$ & -0.3130 & $(0.6391)$ & -0.0499 & $(0.1328)$ \\
\hline $56-65$ years & -0.2583 & $(0.1481)$ & -0.3577 & $(0.7751)$ & -0.1479 & $(0.1555)$ \\
\hline East Germany & 0.3877 & $(0.2290)$ & 0.5572 & $(0.7366)$ & 0.1742 & $(0.2299)$ \\
\hline \multicolumn{7}{|l|}{ Initial condition } \\
\hline poverty status in 1991 & 0.6065 & $(0.0639)$ & & & 0.3903 & $(0.0601)$ \\
\hline \multicolumn{7}{|c|}{ Random effect (time averages) } \\
\hline employed & -0.4020 & $(0.0924)$ & & & -0.2742 & $(0.0954)$ \\
\hline living w. others & 0.0864 & $(0.1266)$ & & & 0.0883 & $(0.1235)$ \\
\hline university degree & -0.4179 & $(0.1484)$ & & & -0.4108 & $(0.1538)$ \\
\hline Abitur or Lehre ${ }^{d}$ & -0.1428 & $(0.1023)$ & & & -0.0950 & $(0.0940)$ \\
\hline $26-35$ years & 0.5746 & $(0.1957)$ & & & 0.4895 & $(0.1724)$ \\
\hline $36-45$ years & 0.5454 & $(0.1819)$ & & & 0.4490 & $(0.1657)$ \\
\hline $46-55$ years & 0.3835 & $(0.2080)$ & & & 0.2503 & $(0.1907)$ \\
\hline $56-65$ years & 0.3655 & $(0.2224)$ & & & 0.2542 & $(0.2092)$ \\
\hline non-German nationality & 0.3505 & $(0.0682)$ & & & 0.3203 & $(0.0596)$ \\
\hline East Germany & -0.0988 & $(0.2344)$ & & & 0.0606 & $(0.2348)$ \\
\hline constant & 1.1815 & $(0.1689)$ & & & -1.3682 & (0.1398) \\
\hline
\end{tabular}

Source: GSOEP, 1991 - 2001, balanced sample of 2427 prime-aged males (18 to 65 years).

${ }^{a}$ Coefficients were rescaled by $\left(1+\hat{\sigma}_{a}^{2}\right)^{-1 / 2}$ to ensure comparability with pooled model.

The estimate of $\sigma_{a}$ was $\hat{\sigma}_{a}=0.7397$ with estimated standard error 0.0414 .

${ }^{b}$ See Honoré/Kyriazidou (2000).

${ }^{c}$ Standard errors account for clustering of observations at individual level.

${ }^{d}$ High-school degree and/or vocational training. 
Table 2. Joint dynamic model of poverty status, employment status and household composition (standard errors in parentheses)

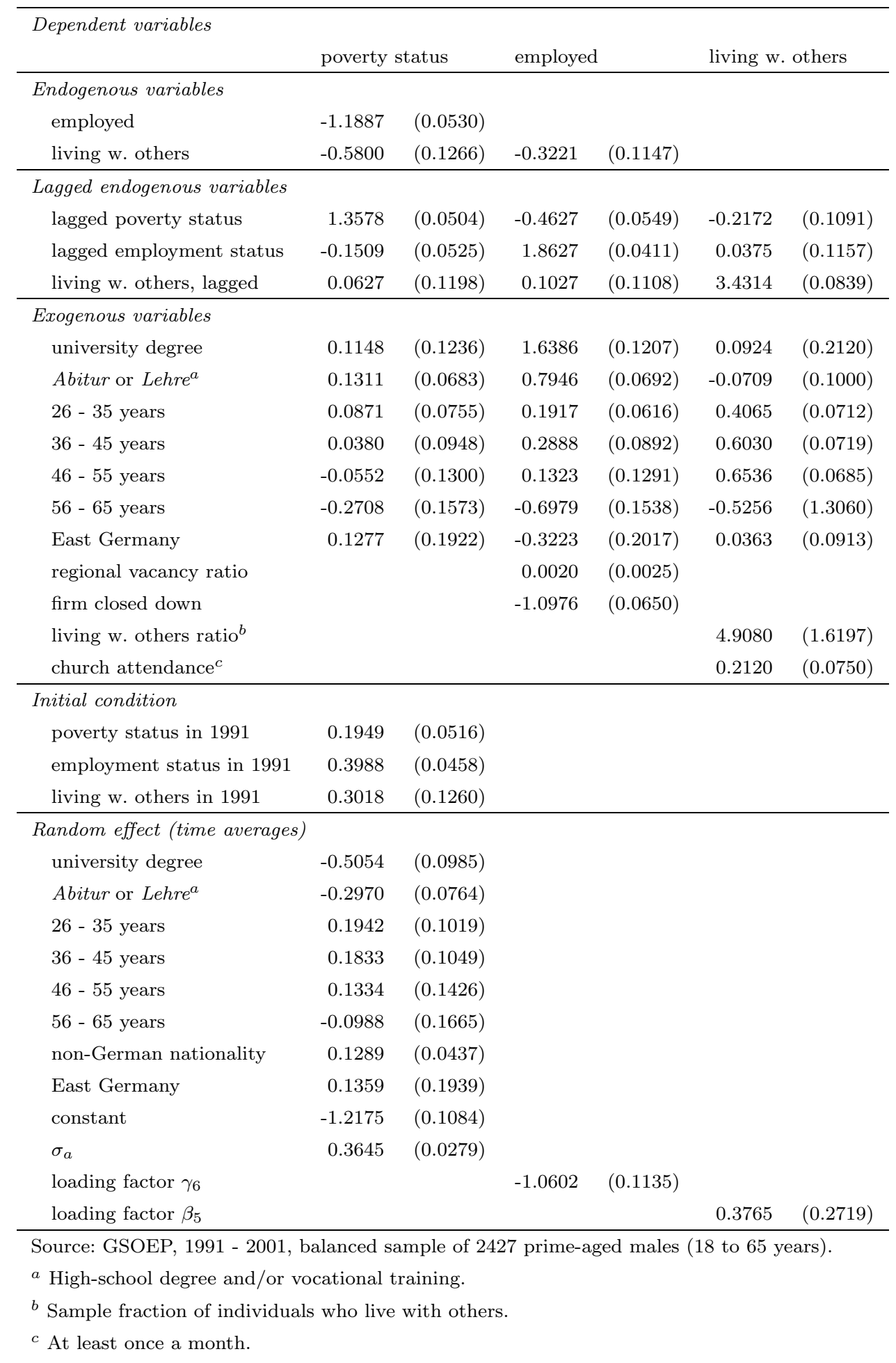


Table 3. State dependence and feedback effects

(average partial effects in percentage points, evaluated for 2001)

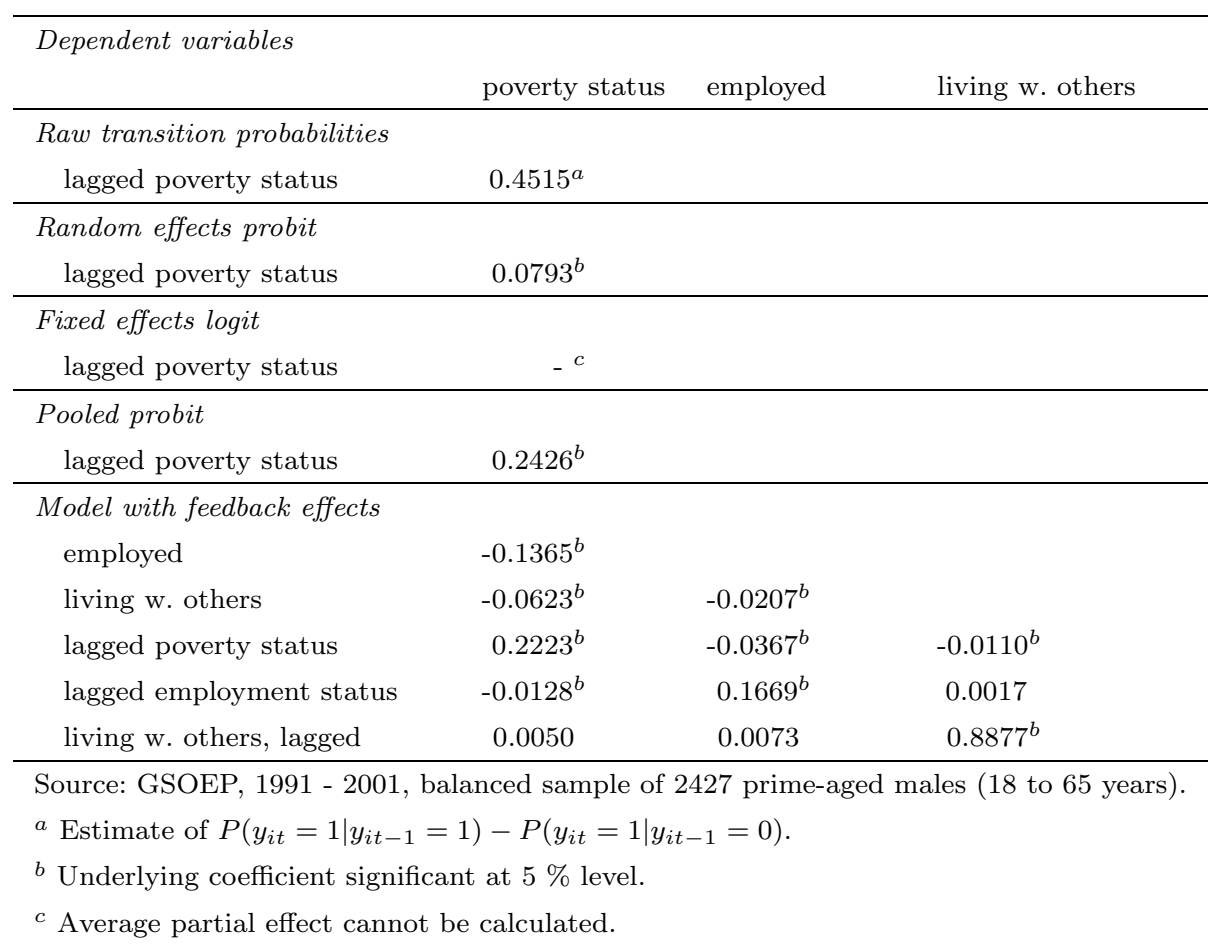

